mit ihr identisch war. Da der Nachweis bei den Thylakoidpräparaten aus beiden Bakterien sehr deutlich ausfiel, obwohl elektronenoptisch keine Verunreinigungen mit Zellwänden nachzuweisen war, erscheint es sicher, daß diese Substanz in den Thylakoiden vorkommt. Neuraminsäure ließ sich dagegen nicht nachweisen. Ebenso fehlt Heptose.

Wie die vorliegenden Ergebnisse zeigen, enthalten auch bakterielle Thylakoide neben Proteinen und Phospholipiden Kohlenhydrate als Strukturkomponente. Weder die Extraktion mit Methanol-Chloroform noch die Behandlung mit wäßrigem Phenol ergaben eine quantitative Abtrennung der kohlenhydrathaltigen Fraktion. Weitere Untersuchungen

21 O. Lüderitz, A. M. Staub u. O. Westphal, Bacteriol. Rev. 30, 193 [1966]. müssen zeigen, ob die Kohlenhydrate, neben einer Beteiligung an den Phospholipiden als Bestandteile von Lipopolysacchariden oder von Glycoproteinen zu gelten haben. In den zahlreichen, bisher publizierten Strukturmodellen zum molekularen Aufbau der Thylakoide sind neben den Proteinen nur die Sulfo-, Galacto- und Phospholipide berücksichtigt worden. Offensichtlich ist aber die Zahl der an Membranstruktur beteiligten Zucker wesentlich größer als bisher angenommen worden ist.

Die Untersuchungen wurden durch eine Sachbeihilfe der Deutschen Forschungsgemeinschaft unterstützt. Fräulein I. LEUTiger danke ich für ihre gewissenhafte Mitarbeit.

22 G. Drews u. P. Giesbrecht, Arch. Mikrobiol. 53, 255 [1966]; 55, 91 [1966].

\title{
Über die strukturabhängige hämolytische Aktivität einiger Cholin-Phosphatide
}

\author{
D. Arnold und H. U. Weltzien \\ Max-Planck-Institut für Immunbiologie, Freiburg \\ (Z. Naturforsch. 23 b, 675-683 [1968]; eingegangen am 15. September 1967)
}

\begin{abstract}
Eine Anzahl synthetischer Cholinphosphatide wurde hinsichtlich ihrer hämolytischen Wirksamkeit untersucht. Die Lyse der Erythrocyten wurde dabei sowohl in ihrem zeitlichen Verlauf verfolgt, wie auch durch Endpunktsbestimmung bei verschiedenen Konzentrationen quantitativ erfaßt.

Ein direkter Zusammenhang zwischen der Grenzflächenaktivität und der hämolytischen Wirksamkeit der Substanzen besteht nicht. Innerhalb homologer Verbindungsreihen wird dagegen durch die Kettenlänge des apolaren Teils im Molekül eine scharfe Grenze zwischen lytischen und nicht-lytischen Verbindungen gezogen. So zeigt beispielsweise das sehr stark grenzflächenaktive Dipelargonoyl-alecithin gegenüber Humanerythrocyten keinerlei hämolytische Wirksamkeit, während Dicaprinoyl- $\alpha$ lecithin bei gleicher Grenzflächenaktivität eine deutliche Hämolyse verursacht.

Bei einigen der untersuchten Phosphatide wurde überdies ein diskontinuierlicher, zweistufiger Verlauf der Zellzerstörung beobachtet.
\end{abstract}

Eine der bekannten Eigenschaften von Lysophosphatiden ist deren Fähigkeit, Erythrocytenmembranen zu zerstören. KLibansky und De VRIEs ${ }^{1}$ haben die Bindung von Lysolecithin an Erythrocyten in Plasmasuspensionen quantitativ untersucht und die zur Sphärocytose bzw. zur Lyse erforderlichen Konzentrationen bestimmt. GotTFried und RAPPORT ${ }^{2}$ untersuchten die lytische Aktivität verschiedener Plasmalogen-Derivate im Vergleich mit Lysolecithin und versuchten danach eine Klassifizierung der von ihnen untersuchten Substanzen vorzunehmen. Aus den Untersuchungen von Munder, Ferber und FI-

1 Ch. Klibansky u. A. De Vries, Biochim. biophysica Acta [Amsterdam] 70, 176 [1963].

2 E. L. Gottraried u. M. M. Rapport, J. Lipid Res. 4, 57 [1963].
SCHER $^{3}$ kennen wir Zusammenhänge zwischen der hämolytischen Wirksamkeit von Lysolecithin und der Aktivität von Membranenzymen.

Der eigentliche Mechanismus der Cytolyse durch Lysolecithin ist jedoch noch unbekannt. Es scheint uns daher wichtig, über einige in diesem Zusammenhang prinzipielle Fragen mehr experimentelle Daten zu erhalten; so etwa über die Funktion des apolaren und des polaren Anteils eines Lysophosphatides bei der Lyse, über den Einfluß bestimmter Strukturen auf die hämolytische Aktivität, über die Fixierung der Substanzen an der Membran, die Bedeutung

3 P. G. Munder, E. Ferber u. H. Fischer, Z. Naturforschg. 20 b, 1048 [1965]. 
a) Lysolecithin aus Lecithin ex ovo: LL

b) 1-Palmitoyl-glycerin-phosphorsäure-(3) -monocholinester
$\mathrm{CH}_{2}-\mathrm{OCO}-\mathrm{C}_{15} \mathrm{H}_{31}$
(Palmitoyl-lysolecithin) :

$\mathrm{CHOH}$

$\mathrm{CH}_{2}-\mathrm{O}-\mathrm{P}-\mathrm{N} * \mathrm{I}$ * L-Form $\quad$ II: $\mathrm{D}$-Form $\quad$ III : DL-Form

c) Homologe Lysolecithine:

IV: DL-Stearoyl-lysolecithin,

V: L-Pentadecanoyl-lysolecithin,

VI: L-Myristoyl-lysolecithin,

VII: L-Tridecanoyl-lysolecithin,

VIII: L-Lauroyl-lysolecithin.

d) Äther-lysolecithine:

IX

$$
\begin{aligned}
& \mathrm{CH}_{2}-\mathrm{O}-\mathrm{C}_{16} \mathrm{H}_{33} \\
& \mathrm{CH}_{2}-\mathrm{O}-\mathrm{P}-\mathrm{N}
\end{aligned}
$$

Glycerin-hexadecyläther-(1)-phosphorsäure-(3) monocholinester (DL)

e) Abgewandelte Lysolecithine (Racemate) :

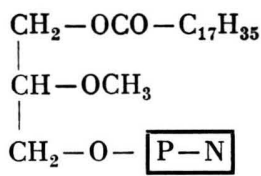

$\mathrm{XI}$

1-Stearoyl-glycerin-methyläther-(2)-phosphorsäure-(3) monocholinester<smiles>CC1=NCOC(COCc2ccccc2)COC1</smiles>

XV

Glycerin-benzyläther-(1) hexadecyläther-(2)-phosphorsäure-(3) -monocholinester

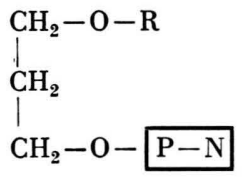

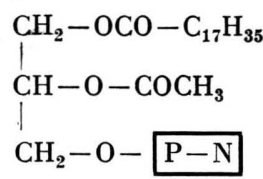

XII

1-Stearoyl-2-acetyl-glycerinphosphorsäure-(3) -monocholinester

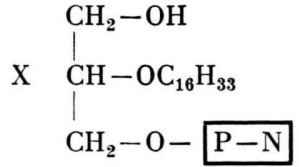

Glycerin-hexadecyläther- (2) -phosphorsäure-(3) monocholinester (DL)<smiles>O=C(OCc1ccccc1)c1ccccc1</smiles>

XIII

l-Palmitoyl-glycerin-benzyläther- (2) -phosphorsäure(3) -monocholinester

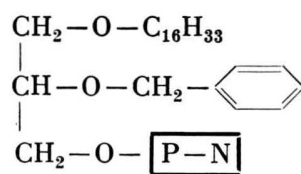

XIV

Glycerin-hexadecyläther-(1) benzyläther-(2) -phosphorsäure-(3)-monocholinester<smiles>[X]C1COC(COC)COc2ccc(cc2)OCC(COC(C)=O)OC1</smiles>

1-( $\omega$-p-Methoxy-phenoxy) -undecanoylglycerin-benzyläther-(2)-phosphorsäure(3) -monocholinester

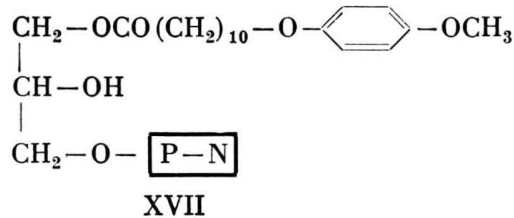

1-( $\omega$-p-Methoxy-phenoxy) -undecanoyl glycerin-phosphorsäure- (3) -monocholinester
Propandiol-alkyläther-(1) -phosphorsäure-(3) -monocholinester, (Äther-desoxy-lysolecithine)

XVIII: $\mathrm{R}=\mathrm{C}_{16} \mathrm{H}_{33}$

XIX: $\mathrm{R}=\mathrm{C}_{14} \mathrm{H}_{29}$

$\mathrm{XX}: \mathrm{R}=\mathrm{C}_{12} \mathrm{H}_{25}$

f) Lecithine (Racemate) :

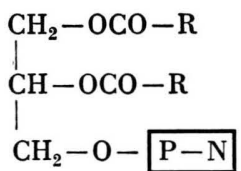

XXI: $\quad \mathrm{R}=\mathrm{C}_{11} \mathrm{H}_{23} \quad$ Dilauroyl- $\alpha$-lecithin

XXII： $\quad \mathrm{R}=\mathrm{C}_{9} \mathrm{H}_{19} \quad$ Dicaprinoyl- $\alpha$-lecithin

XXIII： $\quad \mathrm{R}=\mathrm{C}_{8} \mathrm{H}_{17} \quad$ Dipelargonoyl- $\alpha$-lecithin

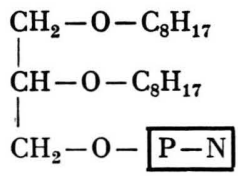

Glycerin-dioctyläther-(1.2)-phosphorsäure(3) -monocholinester

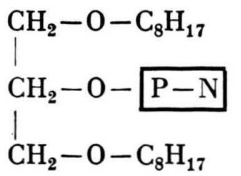

Glycerin-dioctyläther-(1.3) -phosphorsäure

(2) -monocholinester

Abb. 1. Zusammenstellung der untersuchten Verbindungen.

* $-\mathrm{P}-\mathrm{N}=-\stackrel{\ominus}{\mathrm{PO}_{3}}-\mathrm{CH}_{2}-\mathrm{CH}_{2}-\stackrel{\oplus}{\mathrm{N}}\left(\mathrm{CH}_{3}\right)_{3}, \mathrm{H}_{2} \mathrm{O}$ 
monomerer und micellarer Formen oder die Beziehungen zwischen Grenzflächenaktivität und hämolytischer Wirksamkeit. Manche dieser Fragen kann man nur mit einheitlichen, chemisch definierten Substanzen untersuchen. Wir berichten in dieser Arbeit über einige Ergebnisse, die wir über die hämolytische Wirksamkeit einiger synthetischer Cholinphosphatide erhalten haben.

\section{Material und Methoden}

Lecithine und Lysolecithine: Abb. 1 zeigt die Strukturformeln der von uns getesteten Verbindungen. Die Synthese dieser Stoffe haben wir schon früher beschrieben ${ }^{4-7}$. Lediglich die Verbindungen XVI - 1-( $\omega-p$. Methoxy-phenoxy) -undecanoyl-glycerin-benzyläther-(2)phosphorsäure-(3)-monocholinester - und XVII 1-( $\omega$-p-Methoxy-phenoxy)-undecanoyl-glycerin-phosphorsäure-(3)-monocholinester - wurden neu hergestellt. Wir arbeiteten dabei in Analogie zu der für die Lysolecithinsynthese beschriebenen Syntheseweise ${ }^{5}$. Die $\omega-p$ Methoxy-phenoxy-undecansäure wurde nach Vorschriften von ISELE und LütTringhaus ${ }^{8}$ hergestellt.

Analyse für XVI: $\mathrm{C}_{33} \mathrm{H}_{54} \mathrm{NO}_{10} \mathrm{P}(655,78)$

Ber. C $60,44 \quad \mathrm{H} 8,30$ N 2,14 P 4,72,

Gef. C 60,14 H 8,56 N 2,28 P 4,52.

Analyse für XVII: $\mathrm{C}_{26} \mathrm{H}_{48} \mathrm{NO}_{10} \mathrm{P}(565,66)$

Ber. C 55,21 H 8,55 N 2,48 P 5,48,

Gef. C 55,49 H 8,66 N 2,38 P 5,28 .

Lysolecithin aus Lecithin ex ovo bezogen wir von der Nutritional Biochem. Corp. (Cleveland, Ohio).

Grenzflächenaktivität: Die Grenzflächenspannung der Substanzlösungen in $\mathrm{Pill}$ e m e r - Puffer ${ }^{9}$ gegen Luft wurde bei $37^{\circ}$ nach der Ringmethode ${ }^{10}$ gemessen. Es wurden Messungen bei verschiedenen Konzentrationen in $10 \mathrm{ml}$ Lösung in Glasgefäßen von $4 \mathrm{~cm}$ Durchmesser durchgeführt. Um konstante und reproduzierbare Werte zu erhalten, ließen wir die Lösungen vor der Messung jeweils 45 min bei $37^{\circ}$ stehen. Nach jeder Einzelmessung wurde der Ring mit Wasser, Methanol und Chloroform gespült. Zum Vergleich der Grenzflächenaktivität eignet sich die prozentuale Herabsetzung der Grenzflächenspannung von reinem Pill e m e r - Puffer durch $2 \cdot 10^{-8} \mathrm{Mol}$ Substanz $/ \mathrm{ml}$. In diesem Bereich

${ }^{4}$ H. Eibl, D. Arnold, H. U. Weltzien u. O. Westphal, Liebigs Ann. Chem. 709, 226 [1967].

5 D. Arnold, H. U. Weltzien u. O. Westphal, Liebigs Ann. Chem. 709, 234 [1967].

6 H. U. Weltzien u. O. Westphal, Liebigs Ann. Chem. 709, 240 [1967].

7 H. Eibl, H. van den Bosch, L. L. M. van Deenen u. O. WestPHAL, Liebigs Ann. Chem., in Vorbereitung.

8 G. IsEle, Diplomarbeit, Universität Freiburg 1965.

9 E. A. Kabat u. M. M. Mayer, Experimental Immunochemistry, III. S. 149, Springfield 1961.

10 P. Lecomte de Noux, Z. gen. Physiol. 7, 625 [1925]. ändert sich die Grenzflächenspannung unter unseren Bedingungen bei kleinen Konzentrations-Schwankungen nur noch unwesentlich. Die genauen Werte wurden graphisch interpoliert.

Humanerythrocyten: Humanerythrocyten wurden aus frischen oder nur wenige Tage bei $4^{\circ}$ gelagerten ACD-Konserven entnommen und drei- bis viermal mit $\mathrm{Pillem}$ er-Puffer gewaschen. Alle hier beschriebenen Versuche haben wir in Pillemer-Puffer durchgeführt.

Konzentrations-Abhängigkeit des Hämolysegrades: Wir führten Versuche durch, bei denen der Hämolysegrad in Abhängigkeit von der zugegebenen Substanzmenge nach $3 \mathrm{Stdn}$. Inkubationszeit bei $37^{\circ}$ bestimmt wurde. Die sterile Zellsuspension enthielt bei diesen Versuchen $5,6 \cdot 10^{8}$ Erythrocyten $/ \mathrm{ml}$. Wurden die Zellen aus $3 \mathrm{ml}$ dieser Suspension in $3,6 \mathrm{ml}$ dest. Wasser lysiert, so betrug die Extinktion bei $546 \mathrm{~m} \mu$ in den von uns verwendeten optisch identischen Glasröhrchen 1,000. Mit derselben Suspension wurden sämtliche Substanzen in 5 verschiedenen Konzentrationen getestet. Die Ansätze stellten wir nach folgendem Schema her:

\begin{tabular}{llllll}
\hline Röhrchen Nr. & 1 & 2 & 3 & 4 & 5 \\
\hline Erythrocyten-Suspension [ml] & 3 & 3 & 3 & 3 & 3 \\
Pill e m e r-Puffer [ml] & 0,5 & 0,4 & 0,3 & 0,2 & 0,0 \\
Substanzlösung [ml] * & 0,1 & 0,2 & 0,3 & 0,4 & 0,6
\end{tabular}

Adsorptionstest: $\mathrm{Zu} 3 \mathrm{ml}$ Erythrocyten-Suspension $\left(15 \cdot 10^{8}\right.$ Zellen) gaben wir bei $37^{\circ} 0,5 \mathrm{ml}\left(4,5 \cdot 10^{-8}\right.$ Mol) einer Lösung von XIV. Der gleiche Ansatz wurde in 5 weiteren Röhrchen nach verschiedenen Zeitintervallen hergestellt $(15,30,60,90$ und $150 \mathrm{sec})$. Sämtliche Ansätze wurden danach gleichzeitig in einer Zentrifuge innerhalb von $25 \mathrm{sec}$ auf $3000 \mathrm{Upm}$ gebracht. Als Inkubationszeit berechneten wir die Differenz zwischen diesem Zeitpunkt und der jeweiligen Zugabe von XIV. Nach dem Zentrifugieren gaben wir den Überstand jeweils zum Sediment von $3 \mathrm{ml}$ frischer Erythrocyten-Suspension. Die mit XIV beladenen Zellen wurden dagegen mit $3,5 \mathrm{ml}$ frischer Pufferlösung resuspendiert. Bis zu diesem Zeitpunkt war in keiner Probe Hämolyse eingetreten. Nach $3 \mathrm{Stdn}$. Inkubation der resuspendierten Erythrocyten bei $37^{\circ}$ erfolgte die Hämoglobinbestimmung in der oben beschriebenen Weise.

* Die Substanzlösungen enthielten jeweils $9 \cdot 10^{-8} \mathrm{Mol} / \mathrm{ml}$; sie wurden in den in der Tabelle angegebenen Mengen stets zuletzt in die Teströhrchen gegeben. Wie ersichtlich betrug das Gesamtvolumen in allen Röhrchen $3,6 \mathrm{ml}$; darin waren $16,8 \cdot 10^{8}$ Zellen enthalten. Nach 3 Stdn. Inkubationszeit bei $37^{\circ}$ wurde zentrifugiert und die Menge des ausgetretenen Hämoglobins photometrisch bei $546 \mathrm{~m} \mu$ bestimmt. Zum Vergleich der lytischen Aktivitäten bestimmten wir graphisch aus den Lysekurven diejenige Menge an lytischer Substanz, die unter den genannten Bedingungen $50 \%$ Hämolyse verursacht $\left(\mathrm{L}_{50}\right.$-Dosis). 


\section{Ergebnisse}

\section{Grenzflächenaktivität ${ }^{\oplus}$}

\begin{tabular}{cccc}
\hline Substanz Nr. & $\begin{array}{c}\text { Grenzflächen- } \\
\text { Aktivität }\end{array}$ & Substanz Nr. & $\begin{array}{c}\text { Grenzflächen- } \\
\text { Aktivität }\end{array}$ \\
\hline I & 41,7 & XIV & 30,2 \\
II & 42,2 & XV & 36,1 \\
III & 42,9 & XVI & 35,0 \\
IV & 39,0 & XVII & 12,8 \\
V & 41,7 & XVIII & 41,7 \\
VI & 29,1 & XIX & 38,5 \\
VII & 20,9 & XX & 23,1 \\
VIII & 10,6 & XXI & 25,6 \\
IX & 38,8 & XXII & 61,2 \\
X & 39,1 & XXIII & 59,3 \\
XI & 37,5 & XXIV & 39,6 \\
XII & 41,5 & XXV & 41,8 \\
XIII & 42,2 & LL & 44,3 \\
\hline
\end{tabular}

$\oplus$ Grenzflächenaktivität hier als Herabsetzung der Grenzflächenspannung (in Dyn/cm) von Pille m e r-Puffer/Luft bei $37^{\circ}$ in $\%$ durch $2 \cdot 10^{-8}$ Mol Substanz $/ \mathrm{ml}$.

\section{Hämolyse}

Die Lyse von Humanerythrocyten mit den hier untersuchten hämolytisch wirksamen Cholinphosphatiden bei konstanter Temperatur und konstantem $\mathrm{pH}$ ist eine Reaktion, deren Beginn und zeitlicher Verlauf vom Konzentrationsverhältnis der Reaktionspartner und der chemischen Struktur der lytischen Phosphatide abhängt. Wir konnten ihrem Verhalten nach zwei Gruppen von Substanzen unterscheiden:

Zur ersten Gruppe gehören natürliches Lysolecithin (LL) sowie strukturell wenig abgewandelte Homologe (V), Konfigurationsisomere (I, II und III), Ätherlysolecithine (IX, X) und Ätherdesoxy-lysolecithine (XVIII, XIX). Diese unterscheiden sich qualitativ nicht, wenn man bei $37^{\circ}$ den zeitlichen Ablauf von Lysereaktionen verfolgt, bei denen die eingesetzte Substanzmenge zur Totallyse ausreicht: Nach sehr kurzer Inkubationszeit erfolgt die Zerstörung der Erythrocyten in einer raschen Reaktion.

In der zweiten Gruppe - hierzu gehören Stearoyllysolecithin (IV), die Verbindungen XI und XII mit blockierter Hydroxylfunktion durch $O$-Methylbzw. O-Acetylreste, die Benzyl-lysolecithine XIII, XIV und XV und das hämolytisch wirksame Dicaprinoyl-lecithin (XXII) - beobachtet man eine längere "lag phase“ und eine verminderte Reaktionsgeschwindigkeit. Abb. 2 zeigt den prinzipiell möglichen Kurvenverlauf an vier Beispielen. Aus Abb. 3 geht die Konzentrations-Abhängigkeit der „lag phase" und der Reaktionsgeschwindigkeit hervor.

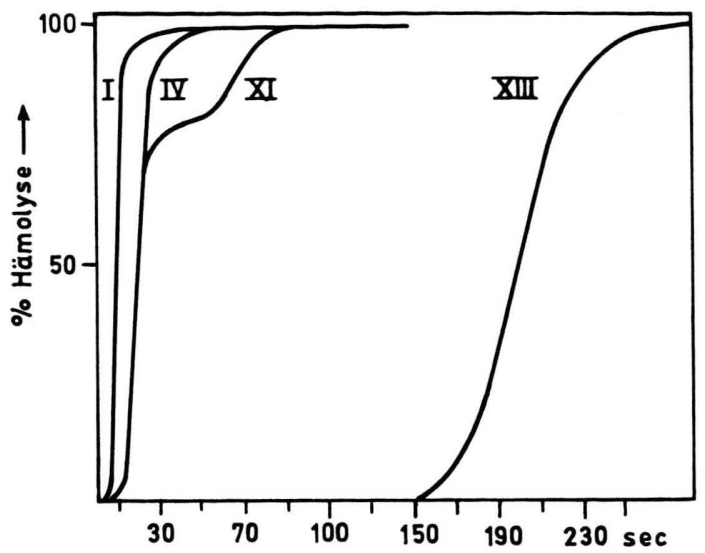

Abb. 2. Lyse von Humanerythrocyten bei $37^{\circ}$ durch verschiedene Lysophosphatide. Konzentration $4 \cdot 10^{-8} \mathrm{Mol} / 7,8$ $\cdot 10^{8}$ Zellen in $2,8 \mathrm{ml} \mathrm{P}$ i $1 \mathrm{l} \mathrm{e} \mathrm{m} \mathrm{e} \mathrm{r} \mathrm{-} \mathrm{Puffer.}$

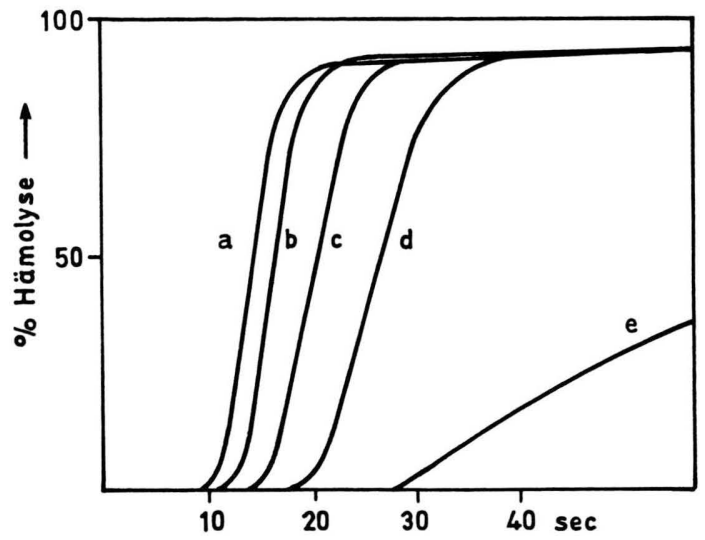

Abb. 3. Lyse von Humanerythrocyten bei $37^{\circ}$ durch verschiedene Konzentrationen von IV. $a=5, b=4, c=2, d=1$, $\mathrm{e}=0,5$-mal $10^{-8} \mathrm{Mol} / 7,8 \cdot 10^{8}$ Zellen in $2,8 \mathrm{ml}$ Pufferlösung.

Das Auftreten einer längeren „lag phase“ scheint die Folge einer geringeren Adsorptionsgeschwindigkeit der betreffenden Substanzen an den Zellen zu sein. Für XIV haben wir dies näher untersucht und eine direkte Abhängigkeit des Hämolysegrades von der Inkubationszeit der Zellen mit lysophosphatidhaltiger Pufferlösung gefunden (vgl. Abb.4).

Mit zunehmender Inkubationszeit fiel gleichzeitig die Restkonzentration an XIV im Überstand stark ab.

In Tab. 2 haben wir die Dauer der "lag phase“ und die Reaktionsgeschwindigkeit im linearen Kurventeil für 2 verschiedene Konzentrationen jeder untersuchten Verbindung angegeben. Die Verbin- 


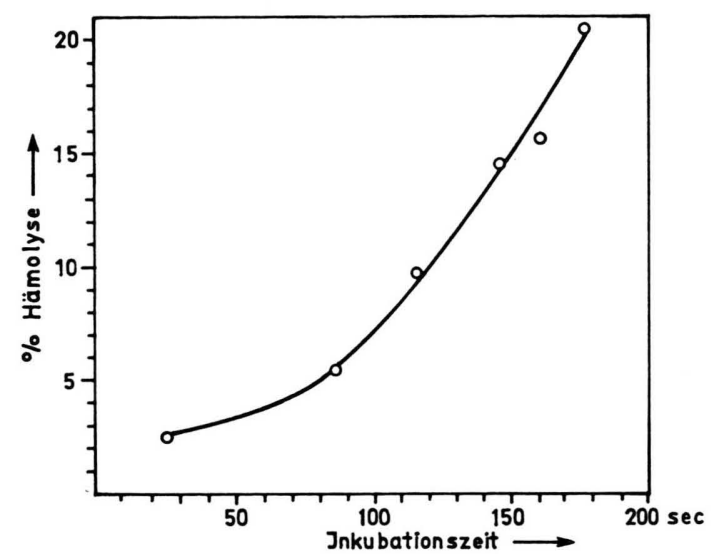

Abb. 4. Adsorptionstest mit XIV. Hämolysegrad von Humanerythrocyten nach $25,85,115,145,160$ und $175 \mathrm{sec}$ Inkubation mit $4,5 \cdot 10^{-8} \mathrm{Mol}$ XIV $\left(1,8 \cdot 10^{7}\right.$ Zellen in $3,5 \mathrm{ml}$ Puffer).

dungen VI, VII, VIII, XVI, XVII, XX, XXI, XXIII, XXIV und XXV waren unter unseren Versuchsbedingungen nicht hämolytisch wirksam.

\begin{tabular}{lcccc}
\hline $\begin{array}{c}\text { Substanz } \\
\text { Nr. }\end{array}$ & \multicolumn{2}{c}{ lag phase [sec] } & \multicolumn{2}{c}{ Geschw. Konstante ** } \\
\cline { 3 - 5 } & $4 \cdot 10^{-8}$ & $2 \cdot 10^{-8}[\mathrm{Mol}]$ & $4 \cdot 10^{-8}$ & $2 \cdot 10^{-8}[\mathrm{Mol}]$ \\
& $*$ & 3 & 21 & 16,5 \\
I & 3 & 3 & 24 & 18,5 \\
II & 2 & 3 & 24 & 22 \\
III & 2 & 17 & 12,5 & 11 \\
IV & 13 & 3 & 21,5 & 1,5 \\
V & 2 & 2,5 & 27 & 26 \\
IX & 2,5 & 2,5 & 27 & 25 \\
X & 2 & 21 & 8,5 & 6,5 \\
XI & 15 & 16 & 11,5 & 7,5 \\
XII & 12 & 200 & 1,5 & 0,8 \\
XIII & 175 & 265 & 1,9 & 0,6 \\
XIV & 195 & 205 & 2 & 1,1 \\
XV & 165 & 8 & 16 & 11 \\
XVIII & 6 & 2,5 & 27 & 25 \\
XIX & 2,5 & 510 & 0,14 & 0,05 \\
XXII & 255 & 7 & 22 & 9 \\
LL & 3 & & & \\
\hline
\end{tabular}

Tab. 2. Zusammenstellung der lag phasen und Reaktionsgeschwindigkeiten für alle untersuchten hämolytischen Phosphatide bei 2 verschiedenen Konzentrationen. * jeweils pro $7,8 \cdot 10^{8}$ Erythrocyten in $2,8 \mathrm{ml}$ Pillemerpuffer (bei $37^{\circ}$ ).

** Anstieg der Lysekurven im linearen Teil (\% Hämolyse/sec).

Bemerkenswert ist der stufenförmige Verlauf der Lysereaktion, wie er aus den Lysekurven für die Substanzen XI und XII ( $O$-Methyl- und $O$-Acetylstearoyl-lysolecithin) hervorgeht. Abb. 5 zeigt die Konzentrations-Abhängigkeit dieses Phänomens für XI.

Die Höhe der Stufen läßt sich durch Änderung der Reaktionstemperatur variieren. So verschwindet die Stufe für XI und XII, wenn man die Lyse-

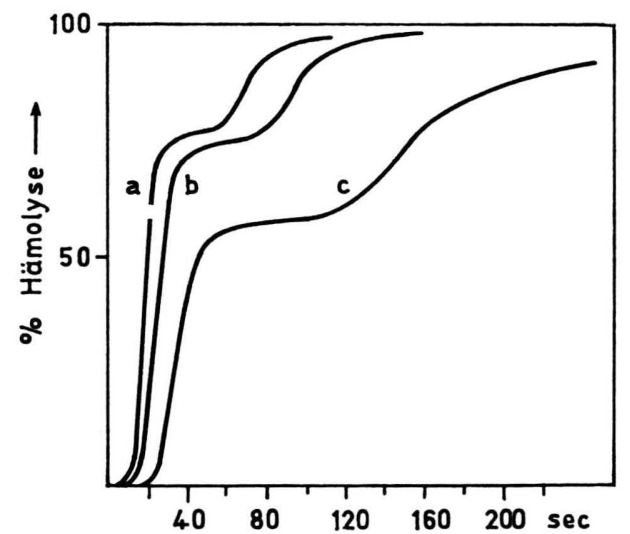

Abb. 5. Stufenweise Hämolyse durch XI bei $37^{\circ}$, Konzentrations-Abhängigkeit. $\mathrm{a}=4, \mathrm{~b}=2, \mathrm{c}=1 \cdot 10^{-8} \mathrm{Mol} \mathrm{XI} / 2,8 \mathrm{ml}$ Puffer $\left(7,8 \cdot 10^{8}\right.$ Zellen).

reaktion bei $10^{\circ}$ durchführt. Bei dieser Temperatur tritt sie jedoch bei den Benzyl-lysolecithinen XIII, XIV und XV sehr ausgeprägt neu auf. Abb. 6 zeigt die Stufenhöhe in Abhängigkeit von der Temperatur, wie wir sie für XV gefunden haben. Außer bei den genannten Verbindungen konnten wir eine

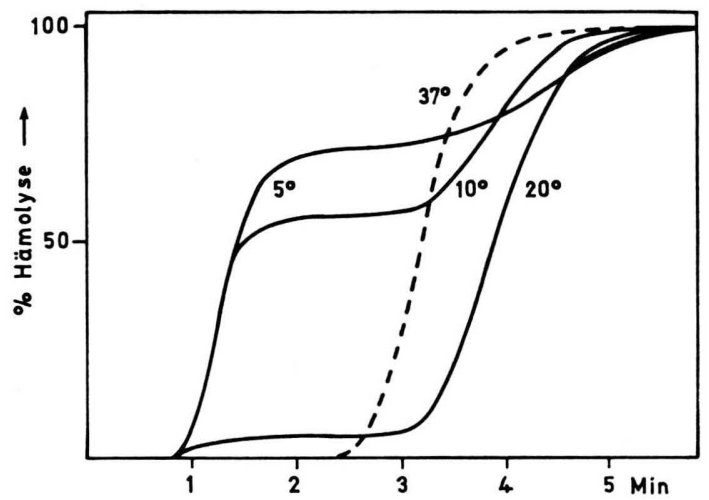

Abb. 6. Stufenweise Hämolyse durch XV, Temperaturabhängigkeit. Konzentration: $4 \cdot 10^{-8} \mathrm{Mol} \mathrm{XV} / 2,8 \mathrm{ml}$ Puffer $(7,8$ $\cdot 10^{8}$ Zellen).

solche Stufe in der Lysekurve allein durch Variation der Reaktionstemperatur nicht erzeugen. Wir erhielten sie jedoch zum Teil, wenn wir verschiedene Substanzen während der Adsorptionsphase von XIII, XIV oder XV zusätzlich auf die Zellen einwirken ließen. Auch dies gelang allerdings nicht mit den Äther-lysolecithinen IX und X. In den Abbn. 7 und 8 sind die verschiedenen Kurven dargestellt, die man erhält, wenn man LL bzw. IV zu unterschiedlichen Zeiten während der lag phase von XIII zugibt. 


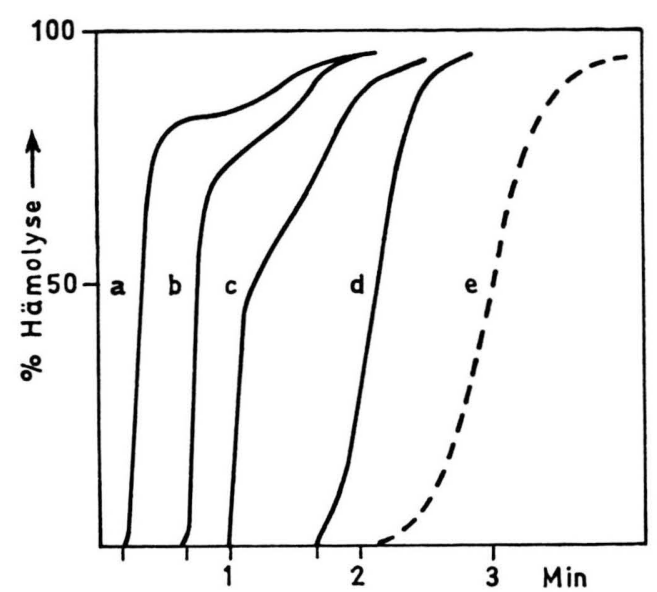

Abb. 7. Hämolysekurven nach Zugabe von $4 \cdot 10^{-8}$ Mol LL während der Adsorptionsphase von $2 \cdot 10^{-8} \mathrm{Mol}$ XIII $\left(7,8 \cdot 10^{8}\right.$ Zellen in $2,8 \mathrm{ml}$ ). Zugabe nach $\mathrm{a}=10, \mathrm{~b}=40, \mathrm{c}=60$, $\mathrm{d}=100$ sec., e: XIII ohne LL. Alle bei $37^{\circ}$.

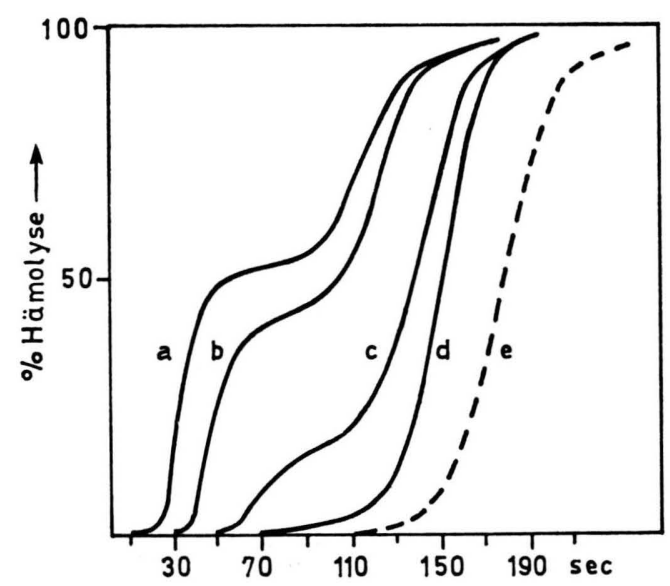

Abb. 8. Hämolysekurven nach Zugabe von $4 \cdot 10^{-8}$ Mol IV während der Adsorptionsphase von $2 \cdot 10^{-8} \mathrm{Mol}$ XIII $\left(7,8 \cdot 10^{8}\right.$ Zellen in $2,8 \mathrm{ml})$. Zugabe nach $\mathrm{a}=10, \mathrm{~b}=30, \mathrm{c}=50$, $\mathrm{d}=70$ sec., e: XIII ohne IV. Alle bei $37^{\circ}$.

Generell reagieren alle untersuchten Verbindungen bei $10^{\circ}$ wesentlich langsamer als bei $37^{\circ}$ (vgl. Tab. 3), wobei das Lecithin XXII eine Sonderstellung einnimmt, da sich hier sowohl die lag phase stark verkürzt als auch die Reaktionsgeschwindig. keit erheblich zunimmt.

Eine Interpretation der genannten Phänomene ist schwierig. Weitere Untersuchungen sollen zeigen, ob bei der Hämolyse zwischen verschiedenen Aggregaten der Lysophosphatide unterschieden wird (z.B. Monomeren und Micellen), oder ob sich in derartigen Stufen ein anderes, allgemeines Prinzip des Lysemechanismus ausdrückt.

\begin{tabular}{lcc}
\hline Substanz Nr. & lag phase [sec] & Geschw. Konstante \\
\hline IV & 30,5 & 7,1 \\
X & 5,5 & 14,5 \\
XI & 27 & 7,8 \\
XIII (1. Stufe) & 65 & 0,69 \\
XIII (2. Stufe) & 245 & 0,68 \\
XV (1. Stufe) & 60 & 1,95 \\
XV (2. Stufe) & 210 & 0,55 \\
XVIII & 11 & 10,5 \\
XIX & 3,5 & 12,3 \\
XXII & 50 & 1,45 \\
LL & 8,5 & 10,9 \\
\hline
\end{tabular}

Tab. 3. Hämolyse bei $10^{\circ}$. Zusammenstellung der lag phasen und Geschwindigkeitskonstanten aus den Lysekurven einiger Phosphatide. Konzentration: $4 \cdot 10^{-8} \mathrm{Mol}$ Substanz $/ 7,8 \cdot 10^{8}$ Zellen in 2,8 ml Pufferlösung.

Bei der Untersuchung der Konzentrations-Abhängigkeit der Hämolyse im Langzeit-Lysetest fanden wir - wie schon Munder, Ferber und Fischer ${ }^{3}$ berichten -, daß der mit einer bestimmten Substanzmenge erreichbare Hämolysegrad von der Inkubationszeit abhängt. Wir können bestätigen, daß der Endwert häufig erst nach mehreren Stdn. erreicht wird, und daß der Einfluß der Inkubationszeit bei tiefen Temperaturen besonders ausgeprägt ist. Bei allzu langer Dauer der Tests wird allerdings die Hämoglobinbestimmung fehlerhaft, da dieses z. T. abgebaut bzw. an den Zellen adsorbiert wird. Alle Daten, die wir in diesen Tests erhielten, müssen von solchen aus den Kurzzeit-Tests deutlich unterschieden werden. Bei jenen beobachteten wir den Vorgang der Hämolyse nur über einen Zeitraum von wenigen min und mußten daher Konzentrationen benutzen, die innerhalb dieses Zeitraums eine möglichst vollständige Hämolyse hervorrufen. Die „hämolytische Aktivität“ unserer Substanzen ließ sich dagegen besser im Langzeit-Test bei nicht zur Totallyse führenden Konzentrationen bestimmen. Unter diesen Bedingungen überlagern sich mehrere Vorgänge, z. B. die direkte lytische Wirksamkeit unserer Substanzen und der Einfluß von Membranenzymen auf die eingesetzten Stoffe oder auf endogene Substrate der Membran ${ }^{3}$. Bei den Kurzzeit-Tests kann dagegen ein Effekt der Membranenzyme vernachlässigt werden. In Abb. 9 wird dies für L-, D- und DL-Palmitoyl-lysolecithin gezeigt; alle 3 Substanzen liefern nahezu identische Lysekurven. Bei den Langzeit-Tests dagegen scheint das nicht metabolisierbare D-Lysolecithin etwas wirksamer zu sein (vgl. Abb. 10). Allerdings kann man wegen der erwähnten Überlagerung verschiedener 
Effekte aus derartigen Testergebnissen nicht unbedingt auf die Metabolisierbarkeit einzelner Substanzen schließen. So erwies sich beispielsweise das Äther-lysolecithin IX unter diesen Bedingungen hämolytisch wirksamer als das Isomere $\mathrm{X}$, obwohl IX nach Untersuchungen von Ferber ${ }^{11}$ zu etwa $50 \%$ enzymatisch zu einem Äther-ester-lecithin acyliert werden kann, $\mathrm{X}$ dagegen nicht.

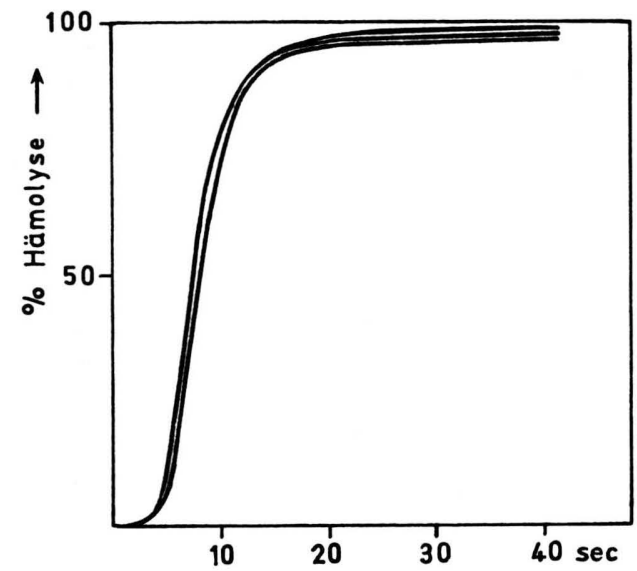

Abb. 9. Kurzzeit-Lysetest mit I, II und III. Lysekurven aufgenommen bei $37^{\circ}$ mit jeweils $1,5 \cdot 10^{-8}$ Mol Substanz pro $7,8 \cdot 10^{8}$ Humanerythrocyten in $2,8 \mathrm{ml}$ Puffer.

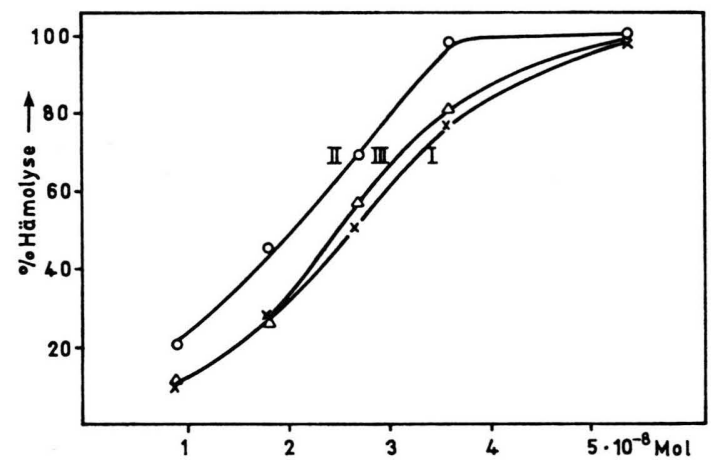

Abb. 10. Langzeit-Lysetest mit I, II und III bei $37^{\circ}$. 1,7 $10^{7}$ Humanerythrocyten in $3,5 \mathrm{ml}$ Puffer jeweils 3 Stdn. mit 5 verschiedenen Konzentrationen von I, II und III inkubiert. Hämoglobinbestimmung im Überstand.

Als charakteristische Größe zum Vergleich der hämolytischen Aktivität verschiedener Substanzen verwendet man vorteilhaft diejenige Substanzmenge (in Mol), welche zu einer 50-proz. Lyse führt $\left(\mathrm{L}_{50}{ }^{-}\right.$ Dosis). Man kann diese Werte graphisch interpolieren, wobei man sich entweder der in Abb. 10 dargestellten Lysekurven bedient oder der nach einer

11 E. FERBER, unveröffentlicht. speziellen logarithmischen Auswertung ${ }^{2}$ erhaltenen Geraden. Die von uns ermittelten $\mathrm{L}_{50}$-Werte sind in Tab. 4 zusammengestellt. Ein kleiner $\mathrm{L}_{50}$-Wert entspricht dabei einer hohen hämolytischen Wirksamkeit. Nicht hämolytisch waren auch hier wieder die Verbindungen VI, VII, VIII, XVII, XX, XXI, XXIV und XXV. XVI und XXIII waren bei der höchsten getesteten Konzentration jeweils schwach hämolytisch wirksam.

\begin{tabular}{cccc}
\hline Substanz Nr. & L $_{\mathbf{5 0}}$-Wert & Substanz Nr. & L $_{\mathbf{5 0}}$-Wert \\
\hline I & 1,57 & XII & 1,25 \\
II & 1,22 & XIII & 1,34 \\
III & 1,51 & XIV & 1,45 \\
IV & 1,24 & XV & 1,45 \\
V & 2,15 & XVIII & 1,71 \\
IX & 1,40 & XIX & 2,83 \\
X & 1,80 & XXII & 2,27 \\
XI & 1,34 & LL & 1,68 \\
\hline
\end{tabular}

Tab. 4. Zusammenstellung der aus dem „Langzeit-Test“ ermittelten $\mathrm{L}_{50}$-Werte (in $10^{-11} \mu \mathrm{Mol} /$ Zelle).

Die hier aufgeführten Zahlenwerte sollten jedoch nur zum Vergleich der untersuchten Verbindungen untereinander benutzt werden. In ihrer absoluten Größe scheinen sie uns dagegen nicht genügend gesichert. So schwankten die Werte bei unseren Versuchen mit ein und derselben Erythrocytensuspension um $\pm 6 \%$. Wurden Zellen aus verschiedenen Konserven verwendet oder an verschiedenen Tagen erhaltene Werte verglichen, so ergaben sich Abweichungen bis zu $10 \%$ vom Mittelwert. Für alle Substanzen ist außerdem der Hämolysegrad in unterschiedlichem Maße vom $\mathrm{pH}$ abhängig. Für LL wurde

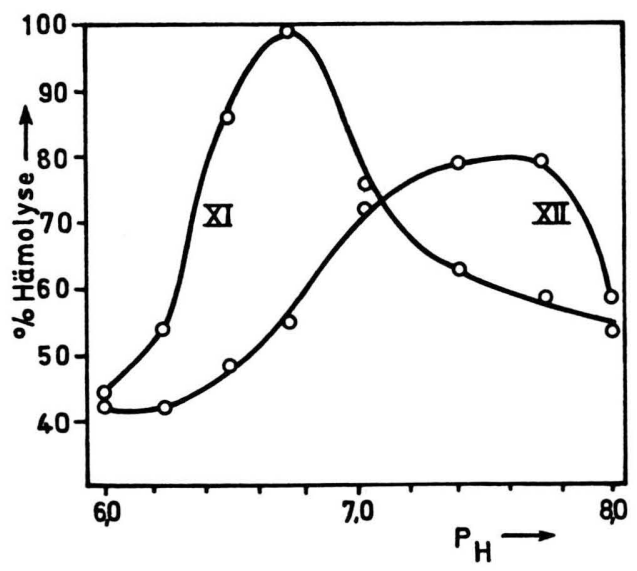

Abb. 11. pH-Abhängigkeit der hämolytischen Wirkung von XI und XII bei $37^{\circ} \cdot 1,7 \cdot 10^{7}$ Humanerythrocyten in $3,5 \mathrm{ml}$ $\mathrm{P}$ ill e m e r-Puffer jeweils 1 Stde. mit $20 \mu \mathrm{g}$ XI bzw. XII inkubiert. 
ein kontinuierlicher Abfall der hämolytischen Aktivität zwischen $\mathrm{pH} 6$ und 8 beschrieben ${ }^{3}$. Es können jedoch bei anderen Substanzen in verschiedenen $\mathrm{pH}$-Bereichen Maxima auftreten, wie wir in Abb. 11 am Beispiel von XI und XII zeigen.

Überdies stellte FERBER ${ }^{11}$ bei Versuchen mit ${ }^{14} \mathrm{C}$ markiertem Lysolecithin fest, daß aus sehr verdünnten Lösungen bis zu 50\% des LL an den Glaswänden der benutzten Gefäße adsorbiert werden kann. Tatsächlich erhielten wir höhere Hämolysewerte, wenn wir vor den Tests die Glasröhrchen mit $10^{-11}-m$. Lösungen der Lysophosphatide und anschließend mit reinem Pillemer-Puffer spülten.

\section{Diskussion}

Wir haben die Grenzflächenaktivität und die hämolytische Wirksamkeit der von uns hergestellten Cholinphosphatide verglichen und keine allgemeine Beziehung gefunden. Zwar sind alle hämolytisch wirksamen Substanzen mehr oder weniger grenzflächenaktiv (mindestens 30\% Herabsetzung der Grenzflächenspannung von reiner Pufferlösung), doch wirken gerade die sehr grenzflächenaktiven kurzkettigen Lecithine XXIII, XXIV und XXV nicht hämolytisch. Bemerkenswert ist daran, daß vor allem die beiden isomeren $\mathrm{Di}-O$-octyl-lecithine XXIV und XXV ausgezeichnete Lösungsvermittler für Lipoide wie Palmitoyl- oder Stearoyl-lecithin sind und in dieser Fähigkeit alle untersuchten langkettigen Lysophosphatide weit übertreffen. Andererseits haben wir für die stark hämolytisch wirkende Substanz XIV, ein Äther-benzyl-lysolecithin, nur eine mäßige Grenzflächenaktivität gefunden.

Betrachtet man die Einflüsse verschiedener chemischer Strukturen, so findet man keine wesentlichen Unterschiede zwischen langkettigen Acyl- oder Alkyllysolecithinen. Auch die freie Hydroxylgruppe scheint nicht von entscheidender Bedeutung zu sein. $O$-methyliertes bzw. $O$-acetyliertes Stearoyl-lysolecithin (XI und XII) kann durchaus mit Stearoyl-lysolecithin selbst (IV) verglichen werden. Eine leichte Abschwächung der lytischen Aktivität ergibt sich bei den Äther-desoxy-lysolecithinen (XVIII). Es ist aber nicht zu entscheiden, ob dies tatsächlich auf den Verlust der Hydroxylgruppe zurückzuführen ist, oder ob man mit der durch Propandiol verlängerten effektiven Kettenlänge evtl. schon ein Optimum der Kettenlänge überschritten hat. Eine solche größere effektive Kettenlänge könnte die Ursache sein, daß Tetradecyl-desoxy-lysolecithin (XIX) noch deutlich hämolytisch wirksam ist, das Myristoyl-lysolecithin (VI) dagegen nicht mehr. Bei der Blockierung der OH-Gruppe mit einem Benzylrest zu den Verbindungen XIII, XIV und XV findet man keine starke Änderung der lytischen Aktivität. Man könnte sich vorstellen, daß die Möglichkeiten zu Wechselwirkungen mit der Zelloberfläche auf Grund van der W a als'scher Kräfte besser werden und dadurch die Vergrößerung des apolaren Teils ausgeglichen wird. Sterische Effekte sollten aber zudem eine Rolle spielen, da einerseits die nach dem Gesichtspunkt von van der Waals- Wechselwirkungen besonders günstige Verbindung XVI nicht hämolytisch wirkt, und andererseits auch bei XIII, XIV und XV Adsorptions- und Reaktionsgeschwindigkeit erheblich verlangsamt werden.

Generell scheint die Kettenlänge der apolaren Alkyl- oder Acylreste im Molekül einen Einfluß sowohl auf den zeitlichen Ablauf wie auch auf die $\mathrm{L}_{50^{-}}$. Werte der Hämolyse zu besitzen. Eine Verkürzung der Kettenlänge gegenüber Palmitoyl-lysolecithin (I) um eine Methylengruppe $\mathrm{zu} \mathrm{V}$ bringt bereits einen Anstieg des $\mathrm{L}_{50}$-Wertes von 1,57 auf 2,15. Die Verbindung VI mit einer um eine weitere Methylengruppe kürzeren Seitenkette ist in dem von uns untersuchten Konzentrationsbereich vollkommen unwirksam. Bei einer Verlängerung der Seitenkette $\mathrm{zu}$ Stearoyl-lysolecithin (IV) dagegen steigt die hämolytische Wirksamkeit an $\left(\mathrm{L}_{50}=1,24\right)$. Im zeitlichen Ablauf der Lysereaktion läßt $\mathrm{V}$ außer einer verstärkten Konzentrations-Abhängigkeit keine Unterschiede zu I erkennen. Eine deutliche (Konzentrations-abhängige) Verzögerung der Hämolyse gegenüber I zeigt sich jedoch bei Stearoyl-lysolecithin (IV): lag phase bei $4 \cdot 10^{-8} \mathrm{Mol} /$ Ansatz $13 \mathrm{sec}$ für IV und 3 sec für I.

Analoge Eigenschaftsänderungen mit der Variation der Kettenlänge findet man bei den Äther-desoxy-lysolecithinen. XIX $\left(\mathrm{C}_{14}\right.$-Kette $)$ reagiert schneller als XVIII $\left(\mathrm{C}_{16}\right.$-Kette), weist aber einen sehr hohen $\mathrm{L}_{50}$-Wert auf $(2,83)$. $\mathrm{XX}\left(\mathrm{C}_{12}\right.$-Kette $)$ ist bereits nicht mehr hämolytisch wirksam.

Bei den Lecithinen ist die Variation der Kettenlänge unter Erhaltung hämolytischer Aktivität in noch geringerem Maße möglich. Caprinoyl-lecithin (XXII) reagiert zwar langsam, ist aber deutlich hämolytisch wirksam $\left(\mathrm{L}_{50}=2,27\right)$. Dagegen finden wir unter unseren Versuchsbedingungen keine lyti- 
sche Aktivität für Pelargonoyl- (XXIII) und Lauroyl-lecithin (XXI). Auf Grund der genannten Ergebnisse muß man also deutlich zwischen Hämolysegrad und Hämolysegeschwindigkeit unterscheiden. Dies wird auch aus folgenden Ergebnissen klar: Bei allen untersuchten Verbindungen mit Ausnahme des Lecithins XXII verlangsamt sich mit sinkender Reaktionstemperatur die Reaktionsgeschwindigkeit, während gleichzeitig die $\mathrm{L}_{50}$-Werte abnehmen, $\mathrm{d}$. $h$. die lytische Aktivität steigt an.

Am schwierigsten zu interpretieren ist der diskontinuierliche Verlauf mancher Hämolysereaktionen. Er könnte mit dem temperaturabhängigen Gleichgewicht zwischen Monomeren und Micellen in der Lösung zusammenhängen. Ebenso könnte diesem Phänomen aber ein zweistufiger Lysemechanismus zugrunde liegen, der möglicherweise ein allgemeines Prinzip der Hämolyse durch Lysophosphatide andeutet, das sich nur bei langsam reagierenden Deri- vaten beobachten läßt. Dafür spräche die Tatsache, daß man durch Zugabe frischer Erythrocyten während des Reaktionsverlaufs mit einer der betreffenden Substanzen eine Kurve mit vier Stufen erzeugen kann, die als unabhängige Überlagerung zweier zweistufiger Lysekurven zu betrachten ist. Es muß jedoch angefügt werden, daß es sich bei den vorliegenden Untersuchungen nur um orientierende Versuche handelt, die ausschließlich mit menschlichen Erythrocyten durchgeführt wurden. Weitere Experimente - auch mit Erythrocyten anderer Species müssen einer endgültigen Interpretation der beobachteten Phänomene vorangehen.

Herrn Prof. Dr. O. Westphal, Herrn Dr. P. G. Munder und besonders Herrn Prof. Dr. H. Fischer danken wir für stetige Unterstützung und wertvolle Diskussionen, Frau Karin Munder für ihre Hilfe bei der Durchführung der Versuche. Die Arbeit wurde zum Teil mit Mitteln der Deutschen Forschungsgemeinschaft durchgeführt.

\title{
Studien zur percutanen Resorption von ${ }^{32} \mathrm{P}$-markiertem DDVP am Rind
}

\author{
Wolfgang Dedek, Heinz Schwarz und Rolf Grahl * \\ Institut für Biophysik der Deutschen Akademie der Wissenschaften zu Berlin, Berlin-Buch, \\ Abteilung Chemische Toxikologie, Leipzig, und Bezirkstierklinik Karl-Marx-Stadt *
}

(Z. Naturforsch. 23 b, 683-686 [1968] ; eingegangen am 26. Atgust 1967)

\begin{abstract}
Die percutane Resorption von ${ }^{32}$ P-markiertem DDVP hoher spezifischer Aktivität aus Lösungen unterschiedlicher Viskosität in Paraffinöl wurde am Rind untersucht (Pour on-Applikation). Im Blut werden kurzzeitig maximale Konzentrationen von $0,3 \mathrm{ppm}$ erreicht, in der Milch sind nach $6-8 \mathrm{~h}$ nur noch $0,005 \mathrm{ppm}$ DDVP nachweisbar. Nicht mehr als $3 \%$ der applizierten Menge an DDVP (50-70 mg pro Tier) verdampfen innerhalb 24 Stunden.
\end{abstract}

Der Phosphorsäureester DDVP (O,O-Dimethyl-dichlor-vinyl-phosphat, Dichlorvos, Vapona ${ }^{\circledR}$, Nuvan $^{\circledR}$ ) wurde erstmalig im Jahre 1951 bekannt und wird seither in ständig zunehmendem Umfang auf Grund seiner vorzüglichen insektiziden Wirkung in der Hygiene und im Pflanzenschutz angewandt ${ }^{1}$. Infolge seines hohen Dampfdruckes wirkt DDVP besonders von der Gasphase aus, zeigt jedoch auch kontaktinsektizide Eigenschaften sowie eine außerondentlich starke cholinergische Wirkung. Es kann in geschlossenen Räumen verwendet werden, da es stark selektive Wirkung aufweist; zahlreiche Insekten werden bereits bei Konzentrationen in der Gasphase abge-

* Anschrift der Verfasser: Dr. rer. nat. habil. W. DEDEK und Dc R. Grahl, X 705 Leipzig, Permoserstraße 15 und Vet.-Rat Dr. H. Schwarz, X 90 Karl-Marx-Stadt, Dresdener Str. 183.

1 G. Schrader, Die Entwicklung neuer insektizider Phosphorsäureester, 3. Auflage, Verlag Chemie, Weinheim 1963, S. $40 \mathrm{ff}$. tötet, bei denen beim Warmblüter, insbesondere beim Menschen, weder eine Hemmung der Cholinesterase noch andere klinische Symptome beobachtet werden können ${ }^{2,3}$. Gasförmiges DDVP wird in Abhängigkeit von der Luftfeuchtigkeit schnell abgebaut ${ }^{3}$; es wird bevorzugt an unterschiedlichen Oberflächen adsorbiert. Zum Einfluß der Gasgeschwindigkeit auf die insektizide Wirkung von DDVP s. l.c. ${ }^{3 a}$. Mit Hilfe von ${ }^{32} \mathrm{P}$-markiertem DDVP konnten Millar et al. ${ }^{4}$ zeigen, daß Fleischwaren bei Lagerung in geschlossenen Räumen maximal 0,25 ppm DDVP aus der Gasphase an der Oberfläche aufnehmen.

2 W. J. Hayes, Bull. Organisat. mond. Santé [Genève] 24, 629 [1961].

3 J. G. Attrield u. D. A. Webster, Chem. and Ind. 1966, 272.

3a D. J. Galley, J. stored Prod. Res. 3, 17 [1967].

4 K. R. Millar u. W. M. Aitken, New Zealand J. Agric. 8, 350 [1965]. 